\title{
Chapter 7. Hypertension complicated by other diseases
}

\author{
Hypertension Research (2009) 32, 51-56; doi:10.1038/hr.2008.11
}

\section{POINT 7A}

Diabetes mellitus

1. The target of blood pressure control in hypertension complicated with diabetes mellitus should be $<130 / 80 \mathrm{~mm} \mathrm{Hg}$.

2. When selecting antihypertensive drugs for diabetic hypertensive patients, an angiotensin-converting enzyme (ACE) inhibitor or angiotensin II receptor blocker (ARB) is recommended as the first choice because of the positive effects on glucose and lipid metabolism and for the prevention of complications, and a $\mathrm{Ca}$ blocker and low-dose of thiazide diuretic should be used concomitantly for blood pressure control. When hypertension is complicated with angina on effort or old myocardial infarction, $\beta$-blockers, which have a cardioprotective effect, can also be used for blood pressure control.

\section{Dyslipidemia}

1. When selecting antihypertensive drugs for hypertension with dyslipidemia, $\alpha$-blockers, ACE inhibitors, Ca channel blockers, and ARBs that improve or do not exacerbate lipid metabolism are considered appropriate.

\section{1) DIABETES MELLITUS}

In diabetic patients, blood pressure should be measured in both a recumbent and standing as well as a sitting position, because orthostatic hypotension is observed in some patients. The frequency of hypertension is about two times higher in diabetic than in nondiabetic individuals according to results in Japan. ${ }^{477}$ In addition, the frequency of diabetes mellitus is two to three times higher in hypertensive patients, ${ }^{477}$ and an etiological relationship between the two diseases has been suggested, that is, type II diabetes and hypertension are major factors of metabolic syndrome (discussed later), having insulin resistance as a common background factor.

Microvascular complications of diabetes mellitus include nephropathy, neuropathy and retinopathy. These conditions may not only cause severe impairment of physical functions and quality of life (QOL) but also affect survival. Both diabetes mellitus and hypertension are important risk factors for macrovascular disease due to atherosclerosis, and the frequencies of cerebrovascular disease and ischemic heart disease are known to be markedly elevated by their concurrence $^{478}$ Therefore, strict control of blood pressure as well as blood glucose level is important for the prevention and treatment of micro- and macrovascular diseases.
With regard to the blood pressure control level in hypertensive patients with diabetes mellitus, the risk of cardiovascular events was significantly reduced in a group managed with a target diastolic pressure of $\leqslant 80 \mathrm{~mm} \mathrm{Hg}$ compared with a group managed with a target of $\leqslant 85$ or $\leqslant 90 \mathrm{~mm} \mathrm{Hg}$ in HOT, ${ }^{139}$ in which antihypertensive treatment was performed primarily using Ca channel blockers. Moreover, the results of UKPDS39 showing that the risk of macro- and microvascular diseases was markedly reduced by lowering mean blood pressure from $157 / 87$ to $147 / 82 \mathrm{~mm} \mathrm{Hg}^{479}$ and the results of a clinical trial, which also indicated the usefulness of antihypertensive treatment in normotensive diabetic patients, ${ }^{480}$ suggest that the therapeutic effect can be increased by setting a lower target of blood pressure control for hypertension complicated with diabetes mellitus. On the basis of these results, the JNC-VI, 1999 WHO/ISH Guidelines, and JSH2000 Guidelines recommended the initiation of antihypertensive treatment for diabetic patients with a high-normal blood pressure of $\geqslant 130 /$ $85 \mathrm{~mm} \mathrm{Hg}$. However, the recommendations of the American Diabetic Association (ADA) in 2007, ${ }^{481}$ JNC7 (2003) ${ }^{38}$ and ESH/ESC Guidelines $(2007)^{66}$ set $<130 / 80 \mathrm{~mm} \mathrm{Hg}$ as a target level of blood pressure control by setting aside the category of high-normal blood pressure on the basis of the results of the HOT ${ }^{139}$ and UKPDS39. ${ }^{478}$ In the TannoSobetsu cho Study of Japan, ${ }^{482}$ the mortality rate due to cardiovascular disease was significantly higher in the group with a systolic pressure of $\geqslant 130 \mathrm{~mm} \mathrm{Hg}$ and a diastolic pressure of $\geqslant 80 \mathrm{~mm} \mathrm{Hg}$ than in the group with an optimal blood pressure of $<120 / 80 \mathrm{~mm} \mathrm{Hg}$ in borderline diabetic/diabetic patients. Therefore, the results in Japan also support a target blood pressure of $<130 / 80 \mathrm{~mm} \mathrm{Hg}$ for hypertensive patients with diabetes mellitus. Blood pressure control should be particularly strict in patients with diabetic nephropathy, and $<125 / 75 \mathrm{~mm} \mathrm{Hg}$ should be the target when the urinary protein level is $\geqslant 1 \mathrm{~g}$ per day.

Treatment should be started when blood pressure is $\geqslant 130 / 80 \mathrm{~mm} \mathrm{Hg}$. In diabetic patients with hypertension, nondrug therapies such as weight control and exercise therapy are expected to promote a decrease in blood pressure associated with an improvement in glucose tolerance through increased insulin resistance. Therefore, for hypertensives with diabetes mellitus, strict lifestyle modifications including weight control, exercise, and restriction of salt intake and the simultaneous initiation of antihypertensive medication are the principal treatments. If the target of blood pressure control is expected to be achieved solely through lifestyle modifications in patients with a blood pressure of 130-139/80-89 mm Hg, control through such modifications may be attempted over a period not exceeding 3 months.

In drug therapy treatment for hypertensives with diabetes mellitus, sufficient consideration of the effects of each antihypertensive drug on insulin sensitivity and glucose and lipid metabolism is necessary. 
Diuretics and $\beta$-blockers have been reported to reduce insulin sensitivity and increase the triglyceride level. Furthermore, $\beta$-blockers make symptoms of hypoglycemia in diabetic patients less perceivable, and disadvantages of both drugs with regard to glucose metabolism have been suggested. In contrast, some $\beta$-blockers that reduce the peripheral vascular resistance have been reported to improve insulin resistance with no adverse effect on lipid metabolism. ACE inhibitors, $\mathrm{ARBs},{ }^{483}$ and dihydropyridine Ca channel blockers, which improve insulin sensitivity and exert no adverse effect on lipid metabolism, are recommended from a metabolic point of view. A comparison of the three classes of drugs showed that ARBs and ACE inhibitors were more effective than Ca channel blockers in suppressing the new occurrence of diabetes mellitus, ${ }^{195,198,295}$ suggesting that ARBs and ACE inhibitors have more favorable effects on insulin resistance than Ca channel blockers. Although $\alpha$-blockers improve glucose and lipid metabolism, whether they prevent target organ damage is unclear.

With regard to the preventive effects of various antihypertensive drugs in diabetic hypertensive patients, ACE inhibitors have been shown to prevent declines in renal function and reduce the frequency of transition to dialysis therapy, even in nonhypertensive patients with type I diabetes accompanied by proteinuria. ${ }^{484}$ In type II diabetic nephropathy, the J-MIND ${ }^{485}$ performed in Japan showed that $\mathrm{Ca}$ channel blockers and ACE inhibitors have comparable effects on proteinuria and renal function in patients with diabetic nephropathy, and the UKPDS39 $9^{479}$ indicated that ACE inhibitors and $\beta$-blockers are equally effective in preventing microvascular disease in diabetic hypertensive patients. RENAAL, ${ }^{140}$ IDNT, ${ }^{467}$ IRMA- $2,{ }^{486}$ and MARVAL $^{487}$ also suggested the effectiveness of ARBs for the treatment of type II diabetic nephropathy. In addition, in Japan, the SMART ${ }^{415}$ and INNOVATION ${ }^{416}$ studies also showed the usefulness of ARBs. Thus, ACE inhibitors and ARBs are clearly useful for the management of diabetic nephropathy, and their administration is recommended if microalbuminuria is observed regardless of the presence or absence of hypertension, but caution is needed because many of these treatments are not covered by medical insurance when blood pressure is $<130 / 80 \mathrm{~mm} \mathrm{Hg}$.

In hypertensive patients with diabetes mellitus, $\mathrm{CAPPP}^{488}$ showed the usefulness of ACE inhibitors, and the HOT ${ }^{139}$ and Syst-Eur ${ }^{489}$ studies reported the usefulness of $\mathrm{Ca}$ channel blockers for the prevention of cardiovascular accidents. UKPDS39 $9^{479}$ also indicated that treatment using an ACE inhibitor or a $\beta$-blocker was similarly useful. In IDNT, ${ }^{435}$ ARBs induced a $4 / 3 \mathrm{~mm} \mathrm{Hg}$ greater decrease in systolic pressure/diastolic pressure than did the placebos, and although no difference was noted in the preventive effect against myocardial infarction or stroke, it was significantly more effective in the prevention of congestive heart failure. In addition, Ca channel blockers showed an antihypertensive effect $3 / 3 \mathrm{~mm} \mathrm{Hg}$ greater than the placebos, and although no difference in the preventive effect against heart failure was noted, they showed a significantly stronger preventive effect against myocardial infarction and a more marked preventive effect against stroke. These results suggest a difference between ARBs and $\mathrm{Ca}$ channel blockers in the preventive effect on cardiovascular disease. With regard to the outcome of macrovascular diseases in type II diabetic patients, LIFE ${ }^{196}$ reported that ARBs were significantly more effective than $\beta$-blockers in preventing cardiovascular disease. Thus, ACE inhibitors, ARBs and Ca channel blockers have been confirmed to be useful for the prevention of cardiovascular accidents in diabetic hypertensive patients. As for a comparison between ACE inhibitors and $\mathrm{Ca}$ channel blockers, their preventive effects were evaluated in the smaller studies $\mathrm{ABCD}^{490}$ and FACET, ${ }^{491}$ and the results suggest that ACE inhibitors are more useful than $\mathrm{Ca}$ channel blockers. However, no difference was observed by subanalysis of ALLHAT, ${ }^{492}$ and further evaluation is necessary to clarify the differences between the effects of ACE inhibitors and Ca channel blockers on macrovascular disease.

With regard to the selection of antihypertensive drugs for diabetic hypertensive patients, ACE inhibitors, ARBs, and long-acting dihydropyridine Ca channel blockers are recommended because of their effects on glucose and lipid metabolism and preventive effects on complications. However, considering evidence with regard to improvements in glucose metabolism and organ protection, a reninangiotensin (RA) system inhibitor (ARB, ACE inhibitor) should be used first, and, if the decrease in blood pressure is insufficient, a $\mathrm{Ca}$ channel blocker or a low dose of a thiazide diuretic should be used in addition as a second choice; if a further decrease in blood pressure is necessary, three drugs should be employed simultaneously. In GUARD, ${ }^{493}$ in which Ca channel blockers and diuretics were compared as a drug to be combined with an RA system inhibitor for the treatment of diabetic nephropathy, combination with a diuretic was more effective for controlling proteinuria, but combination with a $\mathrm{Ca}$ channel blocker was more effective for maintaining the eGFR. In patients with angina on effort or old myocardial infarction, $\beta$-blockers can also be used for blood pressure control because of their cardioprotective effects. Figure 7-1 shows a therapeutic guideline for hypertension with diabetes mellitus.

\section{2) DYSLIPIDEMIA}

As the J-LIT of Japan ${ }^{494}$ has shown, the risk of atherosclerosis clearly increases when hypertension is complicated by hypercholesterolemia, and the aggressive management of both diseases is necessary in such patients. The results of recent clinical studies, including ASCOTLLA, ${ }^{496}$ suggest that the occurrence and recurrence of ischemic heart disease and stroke can be prevented by serum low-density lipoprotein (LDL)-cholesterol-reducing treatment in patients with hypertension with hyper-LDL-cholesterolemia, and the Japanese Guidelines for the Prevention of Atherosclerotic Diseases also propose

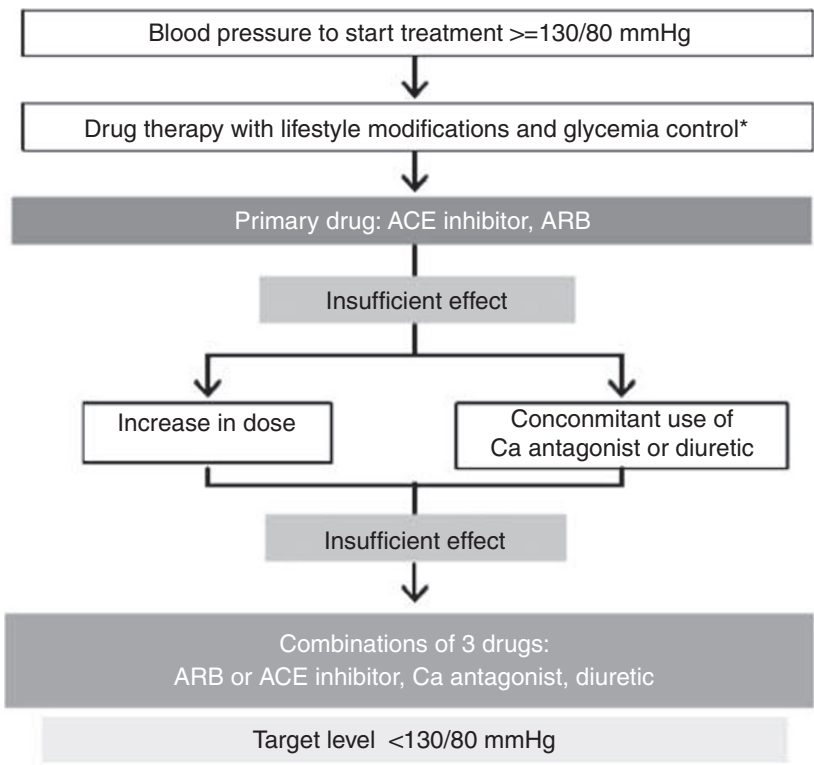

Figure 7-1 Treatment plan for hypertension complicated by diabetes mellitus. *If the blood pressure is $130-139 / 80-89 \mathrm{~mm} \mathrm{Hg}$, and the target of blood pressure control is expected to be achieved through lifestyle modifications, blood pressure control by such modifications may be attempted over a period not exceeding 3 months. 
a stricter control of LDL-cholesterol level if it is complicated by risk factors including hypertension. For patients showing both hyper-LDLcholesterolemia and hypertension, lifestyle modifications, that is, correction of obesity, restriction of saturated fatty acid, cholesterol, and alcohol intake, and an increase in the amount of exercise should be strongly advised. If hypercholesterolemia cannot be resolved by general treatment, drug therapy primarily using an HMG-CoA reductase inhibitor should be performed simultaneously. In patients with hypertension and hypercholesterolemia, the cholesterol level should be controlled to an appropriate therapeutic target level by lifestyle modifications and the use of antihypercholesterolemic drugs. If hypertriglyceridemia or hypo-high-density lipoprotein (HDL)-cholesterolemia is concurrent with hypertension, the presence of insulin resistance or metabolic syndrome should be considered, and this type of dyslipidemia should be corrected by lifestyle modifications and the use of drugs such as fibrates, in principle. If no improvement is noted in dyslipidemia even after practicing lifestyle modifications for 6 months or longer, drugs such as fibrates should be used to correct it.

Concerning the target of blood pressure control in hypercholesterolemic patients, subanalysis of the J-LIT ${ }^{123}$ showed that the frequency of cardiovascular diseases increased significantly when blood pressure was $\geqslant 130 / 80 \mathrm{~mm} \mathrm{Hg}$ in a hypercholesterolemic group with a total cholesterol level of $\geqslant 220 \mathrm{mg} \mathrm{dl}^{-1}$ under statin administration, whereas it increased significantly when blood pressure was $\geqslant 140 / 90 \mathrm{~mm} \mathrm{Hg}$ in the group with a cholesterol level controlled to within a normal range. If future epidemiological studies yield similar results, the target of blood pressure control for dyslipidemic patients may be lowered. In selecting antihypertensive drugs for dyslipidemic patients, the effects of various antihypertensive drugs on lipid metabolism must be considered. Thiazide and loop diuretics at high doses are known to elevate the serum total cholesterol, triglyceride, and LDL-cholesterol levels, but whether thiazides at a low dose also increase lipids is unclear. $\beta$-blockers have been reported to increase the serum triglyceride level or reduce the HDLcholesterol level. $\alpha$-blockers reduce the serum cholesterol level and increase the HDL-cholesterol level. ACE inhibitors, ARBs, Ca channel blockers, and central sympathomimetic drugs have no effect on serum lipid levels.

For hypertensive patients with dyslipidemia, antihypertensive drugs that improve or have no adverse effect on, lipid metabolism, such as $\alpha$-blockers, ACE inhibitors, Ca channel blockers, and ARBs, are desirable from a metabolic point of view.

\section{POINT 7B}

Obesity

1. Hypertension accompanied with obesity is treated by drug therapy as well as by dietary and exercise therapies to control body weight. Antihypertensive drugs should be selected according to the characteristics of their effects on metabolism, and ARBs and ACE inhibitors are recommended.

\section{Metabolic syndrome}

1. Metabolic syndrome is also an important factor responsible for cardiovascular disease in Japan. Hypertension in patients with metabolic syndrome should be treated with attention to the correction of abdominal obesity and insulin resistance, and ARBs and ACE inhibitors are recommended.

\section{3) OBESITY}

The frequency of hypertension is reported to be two to three times higher in obese than nonobese individuals. ${ }^{496}$ In particular, excessive weight gain from a young age is an important risk factor for hypertension. The sympathetic nervous system, sodium retention/ salt sensitivity and insulin resistance have been suggested to be involved in the etiology of hypertension accompanied by obesity. Sleep apnea syndrome is occasionally observed in obese patients, and it may be a factor involved in the progression of hypertension.

In treating hypertension, risk factors for cardiovascular disease, which often complicate obesity, must be reduced. Weight control through dietary and exercise therapies should be attempted first, but, if the decrease in blood pressure is insufficient even after guidance in weight control, drug therapy should be introduced. It is practical to select antihypertensive drugs on the basis of characteristics other than antihypertensive effect, and ACE inhibitors and ARBs are recommended because of their favorable effects on abnormal glucose metabolism and insulin resistance. In CASE-J, ${ }^{195}$ a large clinical study performed in Japan, the new occurrence of diabetes mellitus was significantly lower in the group treated with the ARB candesartan even in the case of obesity. Thiazide diuretics exert no marked effect on metabolism if administered at half the routine dose. Hypertension with obesity is often resistant to treatment, and the concomitant use of a thiazide diuretic is useful in such patients.

\section{4) METABOLIC SYNDROME}

The concurrence of hypertension, dyslipidemia (hypertriglyceridemia, hypo-HDL-cholesterolemia), obesity and abnormal glucose metabolism has been shown by many epidemiological studies to synergistically increase the risk of atherosclerotic diseases, including ischemic heart disease. As insulin resistance is involved as a common background factor in these risk factors of atherosclerotic diseases, the condition has been referred to by various names including multiple risk factor syndrome, insulin resistance syndrome and visceral fat syndrome, but the term 'metabolic syndrome' proposed by the National Cholesterol Education Program (NCEP) ATP-III (2001) has recently become universal. ${ }^{497}$ The diagnostic criteria of the NCEP-ATP-III for metabolic syndrome are a high blood pressure $(\geqslant 130 / 85 \mathrm{~mm} \mathrm{Hg}$ ), abnormal glucose tolerance (fasting blood glucose level $\geqslant 110 \mathrm{mg} \mathrm{dl}^{-1}$ ), visceral fat type obesity (waist circumference $\geqslant 102 \mathrm{~cm}$ for men, $\geqslant 88 \mathrm{~cm}$ for women), hypertriglyceridemia $\left(\geqslant 150 \mathrm{mg} \mathrm{dl}^{-1}\right)$, and hypo-HDL-cholesterolemia ( $<40 \mathrm{mg} \mathrm{dl}^{-1}$ for men, $<50 \mathrm{mg} \mathrm{dl}^{-1}$ for women) and a condition involving three or more of these five risk factors is regarded as metabolic syndrome.

Diagnostic criteria in Japan were proposed in April 2005 by a joint committee of eight related scientific societies, including The Japanese Society of Hypertension. ${ }^{126}$ According to the criteria shown in Table 7-1, hypertension in metabolic syndrome is accompanied by abdominal obesity concurrent with either abnormal glucose or abnormal lipid metabolism. The main target organ damages related to metabolic syndrome are cardiovascular disease and diabetes mellitus. In the Tanno-Sobetsu cho Study, the incidence was $1.87^{482}$ and 2.17 times $^{498}$ higher for cardiovascular disease and diabetes mellitus, respectively, in patients with metabolic syndrome. Table 7-2 shows the therapeutic approaches. Treatment for metabolic syndrome differs according to the presence or absence of diabetes mellitus. Without diabetes, antihypertensive drugs are prescribed when the blood pressure is $\geqslant 140 / 90 \mathrm{~mm} \mathrm{Hg}$, and only lifestyle modifications are indicated when it is $130-139 / 85-89 \mathrm{~mm} \mathrm{Hg}$. The target of blood pressure control is $<130 / 85 \mathrm{~mm} \mathrm{Hg}$. If diabetes is present, antihypertensive drug therapy should also be started when the blood pressure is 
Table 7-1 Diagnostic criteria for metabolic syndrome (new criteria prepared jointly by eight societies (April 2005))

- Abdominal fat accumulation
Waist circumference
(Corresponds to a visceral fat area of $\geqslant 85 \mathrm{~cm}$ for men, $\geqslant 90 \mathrm{~cm}$ for women
Two or more of the following in addition to the above.
- Lipid levels
Hypertriglyceridemia
and/or
Hypo-HDL-cholesterolemia
- Blood pressure
Systolic pressure
and/or
Diastolic pressure

Abbreviation: HDL, high-density lipoprotein.

Table 7-2 Treatment for hypertension complicated by metabolic syndrome

\begin{tabular}{cc}
\hline - Diabetes $(-)$ & BP $\geqslant 140 / 90 \mathrm{~mm} \mathrm{Hg}$ \\
& Treatment for hypertension \\
& BP $130-139 / 85-89 \mathrm{~mm} \mathrm{Hg}$ \\
Lifestyle modifications \\
- Diabetes $(+)$ & BP $\geqslant 130 / 80 \mathrm{~mm} \mathrm{Hg}$ \\
& Treatment for hypertension
\end{tabular}

Abbreviation: BP, blood pressure.

Selection of antihypertensive drugs: selected primarily from ARB and ACE inhibitors with a strong insulin resistance-reducing effect.

$\geqslant 130 / 80 \mathrm{~mm} \mathrm{Hg}$ in patients with metabolic syndrome with a target of $<130 / 80 \mathrm{~mm} \mathrm{Hg}$. The aim of treatment is the correction of abdominal obesity through dietary and exercise therapies. If antihypertensive drugs are used, ARBs, ACE inhibitors, Ca channel blockers and $\alpha$-blockers that improve insulin resistance are desirable. Control of the new occurrence of diabetes mellitus is related to the alleviation of insulin resistance, and this relationship has been established in clinical studies using ARBs and ACE inhibitors. Although Ca channel blockers have also been shown to reduce the occurrence of diabetes, this effect is significantly lower than that of ARBs or ACE inhibitors, as suggested by VALUE, ${ }^{198}$ CASE-J, ${ }^{195}$ and ALLHAT, ${ }^{295}$ so that RA system inhibitors such as ARBs and ACE inhibitors are recommended first. The greater effectiveness of ARBs than Ca channel blockers in obese people shown by CASE-J ${ }^{195}$ also supports this recommendation. However, there is no sound evidence that RA system inhibitors are effective in the prevention of cardiovascular disease in patients with hypertension in metabolic syndrome. ${ }^{295}$

\section{POINT 7C}

Sleep apnea syndrome

1. Sleep apnea syndrome increases with obesity and, being a risk factor for metabolic syndrome, is considered to be a pathological cause of secondary hypertension, which is also expected to increase in Japan.

2. In Japan, sleep apnea syndrome is often observed in nonobese individuals having particular skeletal characteristics of the face, such as micrognathia.

3. In addition to typical patients, who are obese and complain of sleepiness during the daytime, patients who exhibit nocturia, nocturnal dyspnea, cardiovascular events with a night-time onset, resistant hypertension, particularly resistant morning hypertension, and left ventricular hypertrophy despite a normal blood pressure should be suspected of having sleep apnea syndrome.

4. In many patients with sleep apnea syndrome, "non-dipper/ riser' type nocturnal hypertension with changes in blood pressure during nocturnal hypoxic attacks is observed, and high blood pressure is sustained until the morning and detected as morning hypertension.

5. Patients with mild-to-moderate hypertension complicated by severe sleep apnea syndrome should be treated by continuous positive airway pressure (CPAP) first.

6. Strict antihypertensive treatment with a low target level of blood pressure control should be administered with particular attention to the nocturnal blood pressure to minimize an increase in the negative intrathoracic pressure load on the thoracic aorta and heart during sleep.

\section{5) SLEEP APNEA SYNDROME}

Obstructive sleep apnea syndrome (OSAS) is a disease in which hypoxemia occurs periodically during sleep owing to respiratory arrest caused by collapse of the upper airway. It has recently attracted attention as a risk factor for cardiovascular diseases such as ischemic heart disease, heart failure, and cerebrovascular diseases, including silent cerebral infarction, in addition to night time sudden cardiac death. ${ }^{499,500}$ Moreover, OSAS is a causative factor for hypertension and is one of the most frequent causes of secondary hypertension. ${ }^{499}$ As OSAS, which enhances the risk of metabolic syndrome, ${ }^{501-503}$ is also expected to increase in Japan, its appropriate diagnosis and treatment have great significance for the efficient diagnosis and treatment of hypertension.

OSAS increases with obesity, but, in Japan, it is also frequently observed in nonobese individuals with particular skeletal characteristics of the face such as micrognathia. ${ }^{504}$ It is important to suspect OSAS not only in typical obese and hypertensive patients having symptoms such as daytime sleepiness, reduced concentration, depression and snoring but also in patients exhibiting nocturia, nocturnal dyspnea (feelings of suffocation), a history of cardiovascular events with a nocturnal onset (myocardial infarction, stroke, acute aortic dissection, supraventricular or ventricular arrhythmia, etc.), resistant hypertension (particularly resistant morning hypertension) and left ventricular hypertrophy despite normal blood pressure, because hypertensive patients are often asymptomatic (Table 7-3). ${ }^{499,505-508}$ OSAS is diagnosed and staged by sleep polygraphy, and is considered to be mild when the apnea-hypopnea index (number of apneic or hypopneic periods per hour) is $5-15$, moderate when it is $15-30$ and severe when it is $\geqslant 30$.

In OSAS, non-dipper hypertension as well as daytime hypertension is frequently observed, and nocturnal high blood pressure is sustained to the morning and often detected as morning hypertension by home blood pressure measurement. ${ }^{509-511}$ OSAS causes hypertension or an increase in fluctuation of the blood pressure by a wide variety of mechanisms. Activation of the sympathetic nervous system ${ }^{509-512}$ and renin-angiotensin-aldosterone (RAA) system ${ }^{513}$ increases in oxidative stress $^{514,515}$ and inflammatory reactions, ${ }^{516}$ leptin resistance ${ }^{517}$ and insulin resistance ${ }^{518}$ are considered to be involved in a complex manner. Furthermore, in nondipper hypertension with OSAS, marked surges of blood pressure are observed during apneic periods, possibly inducing cardiovascular events with a night time onset. ${ }^{519}$ Recently, an enhancement of the morning surge has been reported in children with 
Table 7-3 Keywords for the detection of obstructive sleep apnea syndrome

\begin{tabular}{ll}
\hline Symptoms & $\begin{array}{l}\text { Daytime sleepiness, reduced concentration, depression, indefinite } \\
\text { complaints (headache, malaise) at awakening or in the morning, } \\
\text { marked snoring, frequent awakening during the night, nocturia, } \\
\text { and nocturnal dyspnea (feelings of suffocation) } \\
\text { Obesity, micrognathia }\end{array}$ \\
$\begin{array}{l}\text { Physical } \\
\text { findings }\end{array}$ & $\begin{array}{l}\text { Resistant morning hypertension (including nocturnal hypertension) } \\
\text { examinations on }\end{array}$ \\
& $\begin{array}{l}\text { Left ventricular hypertrophy (particularly when the clinic and } \\
\text { home blood pressures are normal) } \\
\text { Cardiovascular events (including atrial fibrillation and } \\
\text { ventricular arrhythmias) with a night-time onset } \\
\text { Metabolic syndrome }\end{array}$
\end{tabular}

OSAS who have not developed atherosclerosis. ${ }^{520}$ In OSAS, increases in the vascular responsiveness and chemoreceptor sensitivity due to nocturnal hypoxemia may enhance pressor responses to stimulation such as sympathetic activities, increase changes in the blood pressure and aggravate the cardiovascular risk.

As for treatment, CPAP maintenance should be performed first in patients with mild-to-moderate hypertension complicated by moderate-to-severe OSAS. According to reports to date, blood pressure was lowered, 521,522 nocturnal blood surges were reduced ${ }^{519}$ and cardiovascular prognosis was improved ${ }^{523,524}$ in most patients by CPAP. However, in hypertensive OSAS patients without daytime sleepiness a decrease in daytime blood pressure by CPAP has often been insufficient, and adherence to treatment has been low. ${ }^{525,526}$ As the risk of cardiovascular disease is considered to remain high in hypertensive patients with OSAS with no indication of CPAP or who cannot tolerate more aggressive antihypertensive treatment, particularly with the control of nighttime blood pressure, is recommended to avoid the increase in negative intrathoracic pressure load on the thoracic aorta and the heart during apneic periods (which may reach $80 \mathrm{~mm} \mathrm{Hg}$ ). There is no clear evidence with regard to the selection of antihypertensive drugs for hypertensive patients with OSAS without CPAP. In the evaluation of a small number of patients, a significant decrease in clinical diastolic blood pressure was observed with $\beta$-blockers compared with $\mathrm{Ca}$ channel blockers, ACE inhibitors, diuretics, or ARBs. In addition, although there was no difference in the decrease in daytime blood pressure between patients treated with $\beta$-blockers and those treated with other drugs, the decreases in nighttime systolic and diastolic pressures were significantly greater in those treated with $\beta$-blockers than in those treated with Ca channel blockers, ACE inhibitors or ARBs (no difference compared with diuretics). ${ }^{527}$ However, it is reported that daytime blood pressure could be reduced by treatment with a single antihypertensive drug including $\beta$-blockers, but it was difficult to control nocturnal blood pressure, ${ }^{528}$ and no consensus has been reached with regard to the effectiveness of $\beta$-blockers. For the prevention of target organ damage, RAA system inhibitors are considered to be effective because many patients with OSAS, particularly when it is complicated by obesity, show hyperactivity of the RAA system and left ventricular hypertrophy. In hypertensive OSAS patients, the administration of a diuretic is expected to resolve laryngeal edema and alleviate OSAS. ${ }^{529}$ However, in OSAS patients in whom dry cough is induced by ACE inhibitors, cough may cause inflammation in the upper airway, leading to the exacerbation of OSAS itself. ${ }^{530}$
POINT 7D

Bronchial asthma and chronic obstructive pulmonary disease

1. $\beta$-blockers and $\alpha \beta$-blockers must not be used as antihypertensive drugs in patients with bronchial asthma or chronic obstructive pulmonary disease. ACE inhibitors are not recommended because they may cause dry cough as an adverse effect and increase airway sensitivity. Ca channel blockers, ARBs, and a low dose of diuretics may be used.

\section{Liver diseases}

1. As severe liver dysfunction increases the plasma concentrations of antihypertensive drugs metabolized by the liver, adjustment including a reduction in dose is necessary.

2. $\beta$-blockers may reduce the risk of gastrointestinal bleeding and death in patients with liver cirrhosis. RA system inhibitors may prevent fibrosis of the liver.

\section{6) BRONCHIAL ASTHMA AND CHRONIC OBSTRUCTIVE PULMONARY DISEASE}

In bronchial asthma and chronic obstructive pulmonary disease, not only lung (bronchial) lesions but also fatigue of the respiratory muscles, reduced oxygen transport ability of blood, decline in cardiovascular function, enhancement of sympathetic activity, and activation of the RA system are involved in a complex manner. Although a conclusion has not been reached concerning the problem of whether excessive salt intake increases bronchial sensitivity, ${ }^{531}$ salt restriction at least does not exert an adverse effect on bronchial asthma complicating hypertension. In addition, appropriate exercise guidance depending on the disease type and severity is necessary because exercise may induce asthmatic attacks.

In patients with bronchial asthma or chronic obstructive pulmonary disease or its history, $\beta$-blockers as antihypertensive drugs are contraindicated regardless of whether they are $\beta_{1}$-selective or nonselective, because they may induce airway spasms. In addition, $\alpha, \beta$ blockers are not used because they may induce airway spasms owing to $\beta$-blocking action. ACE inhibitors are known to suppress the occurrence of pneumonia in elderly patients by reducing the threshold of the cough reflex, ${ }^{532,533}$ but they are not recommended for patients with bronchial asthma because they enhance airway sensitivity. ARBs, which belong to RA system inhibitors with ACE inhibitors, may be used because they do not affect airway sensitivity or bronchial smooth muscle. In a study in which ARBs were administered to patients with chronic obstructive pulmonary disease, alleviation of hyperinflation of the lungs and hypoxia-induced hypervolemia was reported. ${ }^{534}$ Both $\alpha_{1}$-blockers and Ca channel blockers reduce the bronchial smooth muscle tone, and bronchial asthma and chronic obstructive pulmonary disease are good indicators. Antihypertensive diuretics should be used at a low dose with appropriate guidance regarding water intake, because they make sputum discharge difficult if they cause dehydration.

Attacks of bronchial asthma are a major cause of stress in patients and increase blood pressure. In addition, $\beta_{2}$-stimulators and steroids, which are used for the treatment of bronchial asthma, may elevate the blood pressure, so that attention to drug interactions is also necessary. In addition, although antihypertensive drugs are considered to cause drug-induced pulmonary disorders less frequently than other drugs, attention must always be paid to their occurrence. 


\section{7) LIVER DISEASES}

Generally, in advanced liver cirrhosis, blood pressure tends to decrease through changes in the hemodynamics and concentrations of physiologically active agents in blood, but usual antihypertensive treatment should be performed if hypertension is present. If edema is noted, there is a possibility of secondary aldosteronism, and attention to changes in the plasma electrolyte concentrations is necessary for using RA system inhibitors or diuretics. The liver is an important organ for drug metabolism, and a marked decrease in the hepatic function due to diseases such as liver cirrhosis may induce a delay in the activation of prodrugs and a rise in the plasma concentrations of drugs metabolized by the liver. As the plasma concentrations of antihypertensive drugs metabolized in the liver may increase in patients with advanced liver cirrhosis, caution such as a reduction of the dose and prolongation of administration intervals is necessary at initial use. Drug-induced hepatopathy owing to labetalol and methyldopa is well known, and these drugs must not be administered to patients with liver dysfunction.

There have been reports of some meta-analyses that noncardioselective $\beta$-blockers such as propranolol lower the risk of gastrointestinal bleeding and death in liver cirrhosis patients by reducing portal pressure. ${ }^{535}$ However, antihypertensive diuretics such as hydrochlorothiazide, chlorthalidone, and furosemide should be used carefully in liver cirrhosis patients, because they may induce hepatic coma through their rapid diuretic action. RA system inhibitors such as ARBs and ACE inhibitors have been suggested by basic studies to prevent fibrosis in the transitional period from chronic hepatitis to liver cirrhosis, but there is no report of results involving a large number of subjects. In nonalcoholic steato-hepatitis (NASH), ARBs are reported to be effective for reversing pathological changes such as fibrosis. ${ }^{536}$

\section{POINT 7E}

Gout/hyperuricemia

1. In hypertension complicated by hyperuricemia, lifestyle guidance such as restriction of energy intake, routine practice of aerobic exercise and restriction of intake of foods and beverages with a very high purine content (for example, beer) should be started at a serum urate level of $\geqslant 7 \mathrm{mg} \mathrm{dl}^{-1}$, and the initiation of urate-lowering drugs with lifestyle modifications should be considered when it is $\geqslant 8 \mathrm{mg} \mathrm{dl}^{-1}$. As for the target of control of the serum urate level during antihypertensive treatment, $\leqslant 6 \mathrm{mg} \mathrm{dl}^{-1}$ should be maintained.

2. Thiazide and loop diuretics, which cause hyperuricemia, should not be used in patients who are likely to develop gout.

3. ACE inhibitors, Ca channel blockers, and $\alpha$-blockers have no adverse effect on urate metabolism. Although ARBs do not affect the urate level, losartan reduces it.

\section{8) GOUT/HYPERURICEMIA}

Urate is the final metabolic product of purine metabolites, and its serum concentration is determined by its production in the body and excretion through the kidney. Urate has a simultaneous antioxidant action and marked vasotoxicity, and hyperuricemia has often been reported to be an independent risk factor for atherosclerosis. However, no large clinical study has shown a reduction of the occurrence of cardiovascular disease through urate-lowering treatment, and so whether urate is a risk factor or disease marker is not clear. As hypertensive patients show reduced urate excretion and increased urate synthesis, reflecting anaerobic metabolism of cells, hypertension is often complicated by hyperuricemia.
The serum urate level in hypertensive patients is correlated with not only the risk of gout but also kidney damage and cardiovascular accidents, though evidence based on an interventional study is lacking. We therefore recommend control of the urate level on the basis of the 6-7-8 rules for comprehensive risk avoidance according to the Guidelines for the Management of Hyperuricemia and Gout ${ }^{537}$ by the Japanese Society of Gout and Nucleic Acid Metabolism. That is, a serum urate level of $\geqslant 7 \mathrm{mg} \mathrm{dl}^{-1}$ is defined as hyperuricemia, and a correction of the serum urate level is started by lifestyle guidance if hypertension is concurrent with hyperuricemia. If the serum urate level still increases or is $\geqslant 8 \mathrm{mg} \mathrm{dl}^{-1}$, urate-lowering therapy should be considered. The serum urate level during antihypertensive treatment should be maintained at $\leqslant 6 \mathrm{mg} \mathrm{dl}^{-1}$. As many patients with hypertension complicated by hyperuricemia have obesity or metabolic syndrome, lifestyle modifications such as restriction of energy intake, routine aerobic exercise, and restriction of intake of foods and beverages with a markedly high purine content (beer, in particular) are necessary. Lifestyle modifications for hypertensive patients, such as restriction of salt intake, should be included in dietary therapy. If the urate level cannot be reduced sufficiently through lifestyle modifications alone, the addition of a urate-lowering drug suited for the type of hyperuricemia should be considered. Urate-lowering drugs include inhibitors of urate synthesis (xanthine oxidase inhibitors) and urate transporter (URAT1) inhibitors, which promote urate excretion. In hyperuricemic patients, the urinary $\mathrm{pH}$ is often low, and it should be adjusted to $\geqslant 6.0$ and $<7.0$ to increase the solubility of urate in urine. If alkalinization of the urine is necessary, fixed combination drugs of sodium bicarbonate or those of Na citrate/K citrate may be administered. The latter are adjusted to reduce the $\mathrm{Na}$ intake compared with sodium bicarbonate, but attention showed be paid to an increase in the serum $\mathrm{K}$ level.

Antihypertensive drugs exert various effects on the urate level. As a rapid decrease in extracellular fluid owing to thiazide or loop diuretics may cause hyperuricemia and induce gout, these drugs should not be used in patients who are prone to developing gout. Potassium-sparing diuretics, such as spironolactone, triamterene and eprelenone, have no adverse effects on urate metabolism. The administration of a $\beta$-blocker at a very high dose increases the serum urate level. A very high dose of an $\alpha, \beta$-blocker also increases serum urate. ACE inhibitors, Ca channel blockers and $\alpha$-blockers have been reported to reduce the serum urate level in some reports but have no effect in others. $\alpha$-Methyldopa has no effect on the serum urate level. With regard to ARBs, some ARBs have been suggested to promote urate reabsorption in the renal tubules, but they exert no clear effect on the serum urate level. Losartan, an ARB, reduces the serum urate level by a mean of $0.7 \mathrm{mg} \mathrm{dl}^{-1}$ by inhibiting the action of URAT1 in the renal tubules. ${ }^{538,539}$ In LIFE, which showed the superiority of the target organ-protective effect of losartan over $\beta$-blockers in severely hypertensive patients, $28 \%$ of the organ-protective effect of losartan, which cannot be explained by its antihypertensive effect alone, was suggested to be derived from an improvement in the urate level. ${ }^{540}$ In fixed combination drugs of ARB and thiazide diuretics, the type of $A R B$ and dose of the diuretic to be combined are adjusted to avoid an excessive increase in the serum urate level.

\section{Citation Information}

We recommend that any citations to information in the Guidelines are presented in the following format:

The Japanese Society of Hypertension Guidelines for the Management of Hypertension (JSH 2009). Hypertens Res 2009; 32: 3-107.

Please refer to the title page for the full list of authors. 